\title{
Prevalence of Frailty Syndrome and Chronic Diseases among the Elderly Population: A Hospital-based Study from a Tertiary Care Center
}

\author{
Kalyanasis Ghosh ${ }^{1}$, Jyotirmoy Pal ${ }^{2}$, Arkapravo Hati ${ }^{3}$, Tarun K Paria ${ }^{4}$, Shubhasis Mahato ${ }^{5}$, Moni S Bhattacharjee ${ }^{6}$
}

\begin{abstract}
Aim and objective: This study is aimed to evaluate the prevalence of chronic diseases and frailty which can inform the next generation of models to understand frailty as an emergent property in a complex adaptive system.

Materials and methods: A cross-sectional observational study was planned to evaluate chronic diseases and frailty upon 200 patients of more than 60 years of age. Patients were evaluated for having frailty syndrome by frailty-defining criteria as defined by Fried and colleagues, along with the presence of some chronic diseases.

Results: The mean age of patients was $71.61 \pm 6.65$ years and $76.5 \%$ of the study population were male. Weight loss, exhaustion, low physical activity, slowness, and reduced grip strength were seen among $40,28,11.5,17.5$, and $49.5 \%$ of the patients, respectively. It was observed that $78(39.0 \%), 74(37 \%)$, and 48 (24\%) patients were in nonfrail, prefrail, and frailty group, respectively. Also, $73.5 \%$ of the total study population had at least one chronic disease and $33(16.5 \%)$ patients had more than two chronic diseases. Chronic diseases were mostly present among the frail group.

Conclusion: Frailty syndrome was very common among the elderly population in our study. Hypertension, diabetes, and osteoarthritis were the commonest comorbidities in the geriatric population of our study.

Keywords: Chronic disease, Diabetes, Frailty syndrome, Hypertension.

Bengal Physician Journal (2021): 10.5005/jp-journals-10070-7048
\end{abstract}

\section{INTRODUCTION}

Geriatric medicine is a branch of medical science dealing with clinical, medical, social, preventive, rehabilitative, and psychological aspects of illness in elderly individuals. In India, 60 years and above are considered as the geriatric age-group. Elderly people frequently suffer from chronic diseases, such as hypertension, ischemic heart disease, diabetes mellitus, chronic kidney disease (CKD), osteoarthritis, depression, hypothyroidism, etc., resulting in an increment of morbidity and mortality.

Frailty is defined as a common clinical syndrome in older individuals with reduced physiological reserve and function across various organ systems, resulting in loss of physical and mental performance and decreased ability to cope with everyday or acute stressors. ${ }^{1,2}$ Frailty involves a complex proinflammatory condition characterized by multisystem dysregulation, such as the immunological, neuroendocrine, and neuromuscular systems, leading to altered homeostatic capacity and augmented susceptibility for subsequent morbidity and mortality. ${ }^{3}$

Fried and colleagues proposed phenotypic criteria for frailty syndrome. Those individuals who meet at least three of the five criteria are considered frail, while those individuals who meet two criteria out of five are considered prefrail, a state between robust and frail. ${ }^{4}$ Frailty-defining criteria proposed by Fried and colleagues have been described in Table 1.

So, to keep the elderly healthy apart from chronic diseases, early detection of frailty and detection of prefrailty are necessary. The surrogate endpoint markers are elevated proinflammatory cytokines and chemokines like tumor necrosis factor-a
${ }^{1}$ Department of Internal Medicine, Diamond Harbour Government Medical College, Diamond Harbour, West Bengal, India

${ }^{2-6}$ Department of Internal Medicine, RG Kar Medical College and Hospital, Kolkata, West Bengal, India

Corresponding Author: Arkapravo Hati, Department of Internal Medicine, RG Kar Medical College and Hospital, Kolkata, West Bengal, India, e-mail: hatiarkapravo@gmail.com

How to cite this article: Ghosh K, Pal J, Hati A, et al. Prevalence of Frailty Syndrome and Chronic Diseases among the Elderly Population: A Hospital-based Study from a Tertiary Care Center. Bengal Physician Journal 2021;8(1):3-8.

Source of support: Nil

Conflict of interest: None

(TNF-a), interleukin-6 (IL-6), and acute-phase inflammatory proteins like C-reactive protein $(\mathrm{CRP})^{5-9}$; reduced IGF-1, sirtuins, albumin, leptin, and $\mathrm{DHEAS}^{10}$; and perturbed neutrophil, monocyte, and white blood cell distribution ${ }^{11,12}$ which indicates dysregulation of multiple systems in frailty. Frailty syndrome is itself an independent predictor of mortality as advanced age is a risk factor for various adverse health consequences, including disability, falls, fractures, and delirium, resulting in poor standards of living. The majority of the medical care services like emergency visits and hospitalization are availed by older adults and the healthcare costs of frail adults are manifold higher than their nonfrail counterparts as shown in multiple cohort studies. ${ }^{13-17}$ To sum up, frailty is associated with 
Table 1: Frailty-defining criteria proposed by Fried et al.

Cardiovascular health study

Characteristics of Frailty measure

1. Weight loss (unintentional)/ >10 lb lost unintentionally prior sarcopenia (loss of muscle mass) year

2. Exhaustion/poor endurance "Exhaustion" (self-report)

3. Weakness

4. Low activity

5. Slowness

Grip strength: lowest 20\% (by gender and body mass index)

$\mathrm{kcal} / \mathrm{wk}$ : lowest $20 \%$ males:

$<383 \mathrm{kcal} / \mathrm{wk}$; females:

$<270 \mathrm{kcal} / \mathrm{wk}$

Walking time/15 ft: slowest $20 \%$ (by gender and height)

increased health consciousness, loss of adaptive capabilities of the body and decreased resistance to adverse life events, a decline in living standards, and a decreased chance of survival in older adults. ${ }^{18-20}$

In this study, we have tried to detect and evaluate the prevalence of chronic diseases and frailty in the elderly.

\section{Materials and Methods}

A cross-sectional observational study was planned, after institutional ethics clearance, to evaluate the prevalence of chronic diseases and frailty upon 200 patients of more than 60 years of age attending the Medicine Outpatients Department and Geriatric Clinic of a tertiary care center between April 2018 and September 2019. Patients were evaluated for having frailty syndrome by frailtydefining criteria as defined by Fried and colleagues, along with the presence of some chronic diseases, such as hypertension, ischemic heart disease, diabetes mellitus, osteoarthritis, CKD, depressive disorder, and hypothyroidism.

The following five items were proposed by Fried et al. for consideration of the diagnosis of frailty syndrome, namely:

- Unintentional weight loss: Loss of more than $4.5 \mathrm{~kg}$ of body weight last year was considered as inclusion criteria for this category.

- Exhaustion: This criterion was assessed by a positive response from individuals by asking whether in the last week there was an inability of going out for that individual or everything that had been done by that person last week was an effort or not.

- Physical activity: Minnesota Leisure Time Activity questionnaire was used for the assessment of physical activities where questions were put about walking, jogging, chores (moderately strenuous), swimming, biking, dancing, aerobics, gardening, and exercise cycling. Standardized algorithm was used for the determination of energy expenditure per week (in kilocalories) and it was further stratified by gender.

- Grip strength: Manual dynamometer was applied in the dominant upper limb and each participant was requested to apply the highest force possible in maximum three attempts and the strength was measured. The individuals were considered positive for this category if they were in the Fried criteria for weakness (after adjusting their respective gender and BMI).

- Gait speed: A chronometer is used to calculate the time required to walk a 4.6-meter-long circuit. The individuals who were considered positive were assessed in the Fried-mentioned walking speed (after adjusting their respective heights and genders).
The presence of chronic diseases was assessed on the basis of thorough history taking and the following parameters: blood pressure, geriatric depression scale (short form), complete blood counts, fasting plasma glucose, electrolytes, renal function tests, thyroid function tests, electrocardiogram, and stress testing or coronary angiography reports which were already available with the patients.

\section{Results}

Our study population was 200 and all were more than 60 years of age. The mean age (mean \pm SD) of patients was $71.61 \pm 6.65$ years with a range of $61.00-90.00$ years and the median age was 69 years. A higher proportion of 153 (76.5\%) patients were male. Total 80 (40.0\%) patients were found to have lost their weight. It was found that 56 (28.0\%) patients were having exhaustion. It was observed that 23 (11.5\%) patients were in low physical activity. In this study, 35 patients (17.5\%) out of 200 were having slowness. It was noted that 99 (49.5\%) patients out of 200 were weak (reduced grip strength). It was found that 48 patients $(24.0 \%$ ) out of 200 were frail (meeting three out of five criteria). In this study, it has been seen that 78 (39.0\%), 74 (37\%), and 48 (24\%) patients were in the nonfrail, prefrail, and frailty groups, respectively. Also, $11.6,19.7,22.5,25.6$, and $70.6 \%$ of frail patients were in $61-65,66-70,71-75,76-80$ years, and more than 80 years age-group, respectively. Also, $48.9 \%$ of the total 47 female patients and $16.3 \%$ of the total 153 male patients were found frail. It has been noticed that $73.5 \%$ of the total study population had at least one chronic disease and 33 (16.5\%) patients had more than two chronic diseases. The prevalence of chronic diseases and nonfrail, prefrail, and frail subjects have been represented through two pie diagrams (Figs 1 and 2). The frequency and percentage of chronic diseases along with their distribution among the nonfrail, prefrail, and frail population have been depicted in Table 2. The distribution of the numbers of chronic diseases among the nonfrail, prefrail, and frail population has also been depicted in Table 3.

\section{Discussion}

This study of 200 geriatric patients will add to the available evidence of the prevalence of frailty syndrome and chronic diseases. The age of the study sample ranged from 61 to 90 years. The total study sample comprised $153(76.5 \%)$ males and 47 (23.5\%) females. The age-group

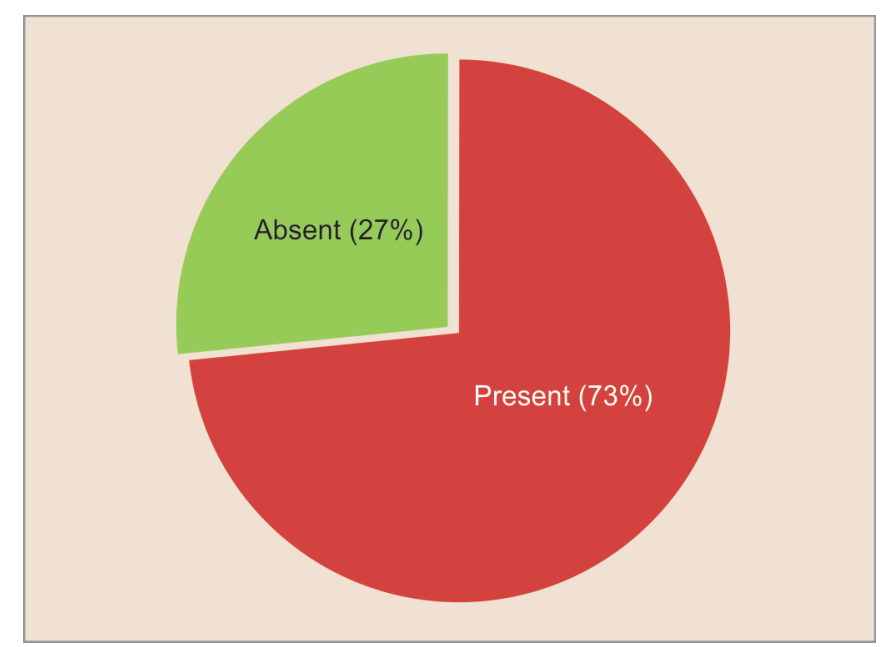

Fig. 1: Pie diagram showing the prevalence of chronic diseases among the study population 


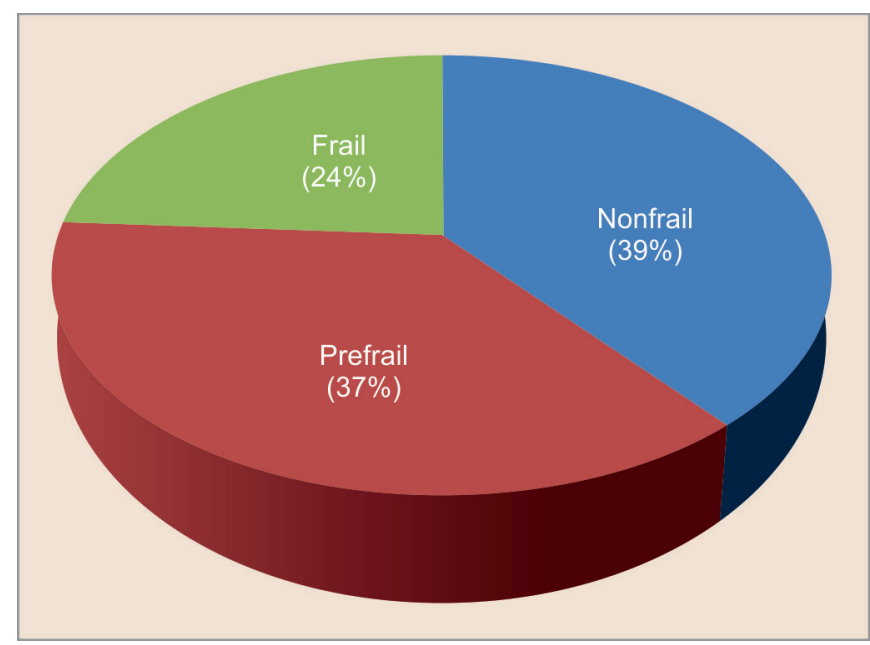

Fig. 2: Pie diagram showing the prevalence of nonfrail, prefrail, and frail among the study population

Table 2: Frequency and percentage of chronic diseases along with their distribution among the nonfrail, prefrail, and frail population

\begin{tabular}{|c|c|c|c|c|}
\hline Chronic diseases & Nonfrail & Prefrail & Frail & $\begin{array}{l}\text { Total } \\
(n=200)\end{array}$ \\
\hline Osteoarthritis & $16(21.6 \%)$ & $24(32.4 \%)$ & $34(46 \%)$ & $74(100 \%)$ \\
\hline Hypertension & $30(27.8 \%)$ & $37(34.2 \%)$ & $41(38 \%)$ & $108(100 \%)$ \\
\hline Diabetes & $19(24.7 \%)$ & $18(23.4 \%)$ & $40(51.9 \%)$ & $77(100 \%)$ \\
\hline $\begin{array}{l}\text { Ischemic heart } \\
\text { disease }\end{array}$ & $10(23.8 \%)$ & $15(35.7 \%)$ & $17(40.5 \%)$ & $42(100 \%)$ \\
\hline $\begin{array}{l}\text { Chronic kidney } \\
\text { disease }\end{array}$ & $0(0 \%)$ & $2(18.2 \%)$ & $9(81.8 \%)$ & $11(100 \%)$ \\
\hline Depression & $1(3.7 \%)$ & $6(22.2 \%)$ & $20(74.1 \%)$ & $27(100 \%)$ \\
\hline Hypothyroidism & $6(19.4 \%)$ & $13(42 \%)$ & $12(38.6 \%)$ & $31(100 \%)$ \\
\hline
\end{tabular}

in $30.5 \%$ of patients was $66-70$ years, $21.5 \%$ was $61-65$ years, $20 \%$ was $71-75$ years, $19.5 \%$ was $76-80$ years, and only $8.5 \%$ of them were more than 80 years of age. The summary of different large studies done previously on frailty has been depicted in Table 4.

Using the data from the cardiovascular health study (CHS), Fried et al. ${ }^{4}$ conducted a study among 5,317 residents of four U.S. communities aged less than 65 years, using the phenotype of weakness. The prevalence of frailty in that specified population was $6.9 \%$ and was associated with African-American ethnicity, low education, low socioeconomic status, poor health status, comorbidities, female sex, chronic noncommunicable diseases, and disabilities. In the $\mathrm{CHS}$, with age, the prevalence of frailty increased from $3.9 \%$ in the 65 to 74 age-group to $25 \%$ in the $85+$ group and was found to be more in women than men (8 vs $5 \%$ ). ${ }^{4}$

Again, one study conducted in France in 2009 has shown a prevalence of $7.0 \%$ frailty among 6,030 people over 65 years of age. ${ }^{21}$ Another survey in 2009 among 7,510 community-dwelling older adults in 10 European countries revealed that the prevalence of frailty ranged from $5.8 \%$ in Switzerland to $27 \%$ in Spain with an overall prevalence of $17 \% .{ }^{22}$ According to a survey (2008) of 7,334 older adults aged 60 or older living in five large Latin-American and Caribbean cities including Bridgetown, Barbados ( $n=1,446)$; Sao Paulo, Brazil ( $n=1,879)$; Santiago, Chile $(n=1,220)$; Havana, Cuba ( $n=1,726)$; and Mexico City, Mexico $(n=1,063)$, the prevalence of frailty varied from 30 to $48 \%$ in women and from 21 to $35 \%$
Table 3: Distribution of the numbers of chronic diseases among the nonfrail, prefrail, and frail population

\begin{tabular}{lcccc}
\hline Chronic diseases & Nonfrail & Prefrail & Frail & Total \\
\hline $\mathbf{0}$ & 35 & 18 & 0 & 53 \\
Row \% & 66.0 & 34.0 & 0.0 & 100.0 \\
Col \% & 44.9 & 24.3 & 0.0 & 26.5 \\
$\mathbf{1}$ & 21 & 22 & 3 & 46 \\
Row \% & 45.7 & 47.8 & 6.5 & 100.0 \\
Col \% & 26.9 & 29.7 & 6.3 & 23.0 \\
$\mathbf{2}$ & 8 & 21 & 8 & 37 \\
Row \% & 21.6 & 56.8 & 21.6 & 100.0 \\
Col \% & 10.3 & 28.4 & 16.7 & 18.5 \\
$\mathbf{3}$ & 12 & 7 & 14 & 33 \\
Row \% & 36.4 & 21.2 & 42.4 & 100.0 \\
Col \% & 15.4 & 9.5 & 29.2 & 16.5 \\
4 & 2 & 3 & 10 & 15 \\
Row \% & 13.3 & 20.0 & 66.7 & 100.0 \\
Col \% & 2.6 & 4.1 & 20.8 & 7.5 \\
$\mathbf{5}$ & 0 & 2 & 7 & 9 \\
Row \% & 0.0 & 22.2 & 77.8 & 100.0 \\
Col \% & 0.0 & 2.7 & 14.6 & 4.5 \\
$\mathbf{6}$ & 0 & 1 & 5 & 6 \\
Row \% & 0.0 & 16.7 & 83.3 & 100.0 \\
Col \% & 0.0 & 1.4 & 10.4 & 3.0 \\
$\mathbf{7}$ & 0 & 0 & 1 & 1 \\
Row \% & 0.0 & 0.0 & 100.0 & 100.0 \\
Col \% & 0.0 & 0.0 & 2.1 & 0.5 \\
TOTAL & 78 & 74 & 48 & 200 \\
Row \% & 39.0 & 37.0 & 24.0 & 100.0 \\
Col \% & 100.0 & 100.0 & 100.0 & 100.0 \\
\hline & & & &
\end{tabular}

in men, which were much higher than their U.S. and European counterparts. ${ }^{23}$

It is quite evident from the previous studies that the prevalence of frailty varies from population to population significantly and no exact reliable data on the worldwide geriatric population of the same can be found.

In this study, the prevalence of frailty was noted to be $24 \%$ among the total 200 patients. Twenty-three women were found to have frailty syndrome (meeting three out of five criteria of Fried's criteria) among the total 47 women in contrast to 25 of 153 men having frailty. Thus, the prevalence of frailty in women was noted to be higher compared to men which corroborates with the results of the previous studies.

But, the prevalence of frailty itself among the total sample size is much higher than the prevalence mentioned in different previous studies. ${ }^{4,21,23,24}$ This particular study is matching partly with the study conducted in 2008 in Latin-American counties. ${ }^{22}$ This variation of prevalence may be due to the reason that all the previous studies were done in a community-based survey and this study was done in the outpatient department in elderly with some ailments or chronic diseases; the chances of frailty increase as the comorbidities increase. ${ }^{4}$

Using Fried's criteria, it was found that half of the patients have a weakness (decreased grip strength), which corroborates with the previous studies except the study conducted in Rio de Janeiro, Brazil, in 2010, in which slowness was the predominant manifestation. The other manifestations in this study in decreasing the order of frequency are weight loss, exhaustion, slowness, and low physical activity. 
Table 4: Summary of different large studies done previously on frailty

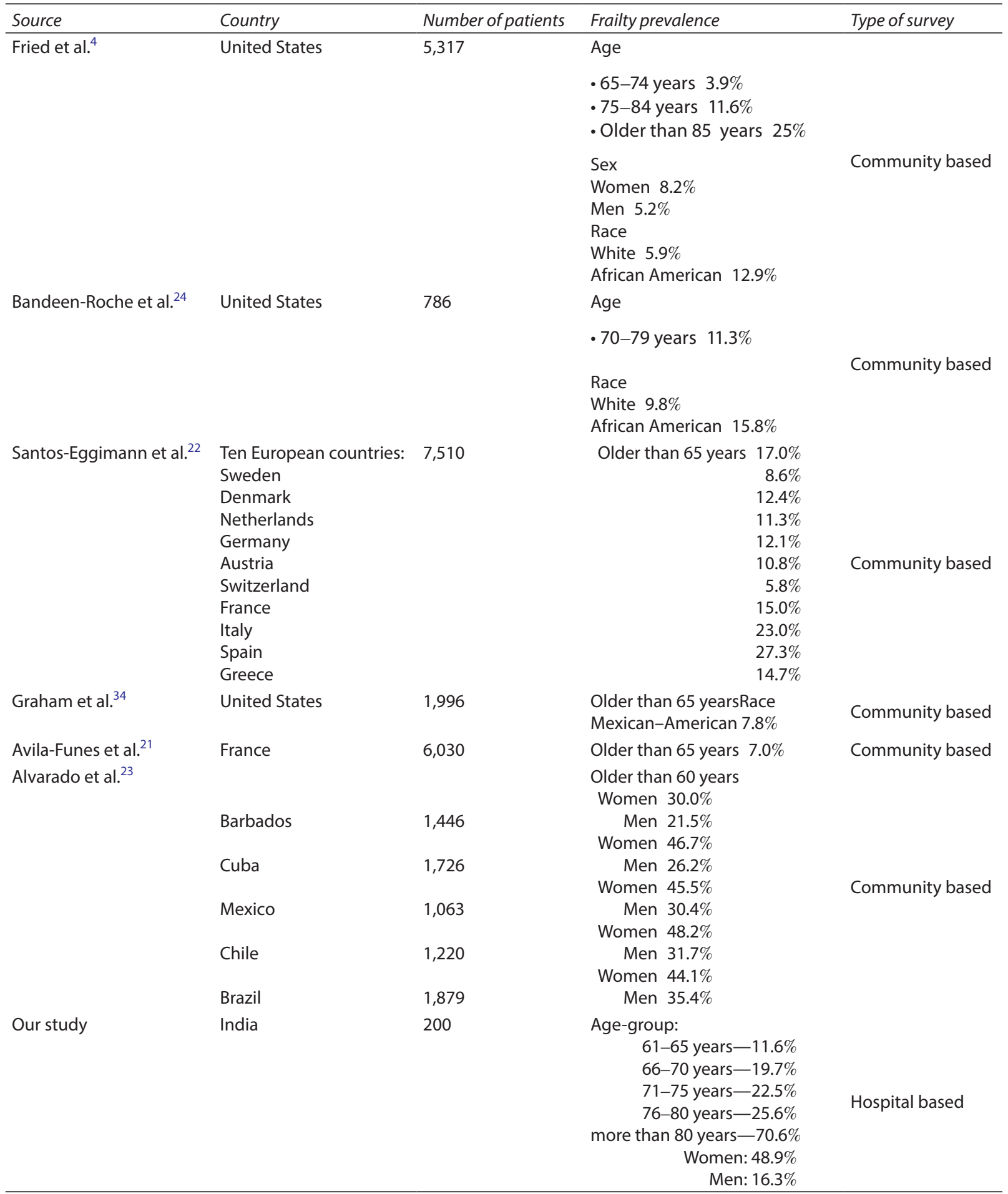

It has been also noted that frailty is prevalent in higher agegroups than lower ones as evident by which is also supported by the previous literatures. ${ }^{4}$

Numerous operational criteria to identify frail elderly persons have been reported in the literature. However, it has been observed great diversity in the composition of the items that compose the instruments used to classify individuals as frail. ${ }^{25}$ For the last 10 years, the concept of frailty phenotype, as proposed by Linda
Fried and coworkers, ${ }^{4}$ has dominated this area of scientific research, and many efforts have been made to confirm the reproducibility and validity of this method. ${ }^{23,26}$ Although this method consists essentially of biological items, some authors believe that the instrument can be affected by other domains, such as cognition. ${ }^{27}$ Moreover, there remains much discussion as to what constitutes the real nature of frailty, what it represents, and whether its content is valid. ${ }^{28}$ For this reason, evaluating different elderly populations 
using these items, testing alternative hypotheses, and reflecting on the meaning of the produced empirical data remain the task of many scientists in the field of human aging.

Studies that have attempted to apply the frailty syndrome-CHS methodology are difficult to compare because there is much variation in the composition and format of the instrument used to measure each item of the scale. ${ }^{21,23,24}$ For example, the "loss of weight" item in the study by Xue and coworkers and the "exhaustion feeling" in the study by Boyd et al. represent such variable items. ${ }^{23,30}$ According to Xue et al., self-reporting may be subject to errors, and BMI may be a better indicator of weight loss. ${ }^{29}$ To verify the feeling of exhaustion, Boyd et al. used one analogical visual scale rather than two questions from the Center for Epidemiological Studies-Depression. ${ }^{30}$

Many health problems are known to increase with age and this demographic trend may lead to an increase in the absolute number of health conditions in the population because there is a growing body of evidence that older people are at risk for multiple comorbid conditions, and thus the healthcare seeking also increases. ${ }^{31}$ The common problems which affect the elderly include arthritis, cataract, ischemic heart disease, diabetes mellitus, hypertension, depression, hypothyroid, CKD, and others.

In this study, seven chronic diseases like hypertension, diabetes mellitus, osteoarthritis, ischemic heart disease, CKD, depression, and hypothyroidism were studied in 200 elderly persons aged more than 60 years of age; of which $73.5 \%$ have one or more chronic diseases.

Hypertension was the most common statistically significant cause of chronic noncommunicable disease morbidity in the studied population which has been buttressed with the reports from other parts of the world. Apart from the age-related cardiovascular changes, aging can lead to an increased sedentary living, overweight and obesity, excessive food intake, and reduced food metabolism which can further predispose the elderly population to hypertension.

Diabetes mellitus was found in $38.5 \%$ of the population which is statistically significant. Moreover, Cigolle et al. had reported that adults with diabetes had an increased prevalence and incidence of geriatric conditions across the age spectrum compared to those without diabetes. ${ }^{32}$

Osteoarthritis was reported in $37 \%$ of the subjects. It has been observed that musculoskeletal disorders lead to incorrect biomechanics, impaired mobility, and skeletal and muscular deconditioning which further results in decreased functional reserves and ability to adapt to physiological, physical, and psychosocial challenges. ${ }^{33}$

Other chronic diseases reported are as follows: ischemic heart disease in $21 \%$, hypothyroidism in $15.5 \%$, depression in $13.5 \%$, and CKD in $5.5 \%$ of the subjects.

More than half $(50.5 \%)$ of the population have two or more chronic diseases. An interesting part of this study is that elderly individuals having frailty syndrome (24\%) have one or more chronic diseases which further strengthens the fact that comorbidities are associated with the frailty syndrome.

\section{Conclusion}

To summarize, a total of 200 elderly persons of more than 60 years of age were observed over a period of 18 months. Out of them, $24 \%$ of them were found to have frailty syndrome (meeting three out of five criteria proposed by Fried and colleagues). Among the study population, women (48.9\%) were found to be more frail than their men $(16.3 \%)$ counterparts, and the prevalence of frailty is more with increasing age. In this study, it has also been observed that $37.5 \%$ of the total study population are in the prefrail group.

In the other part of the study in the same population, it is found that $73.5 \%$ of them are having one or more chronic diseases and the prevalence of some chronic diseases is much more in frailty syndrome like hypertension, CKD, diabetes mellitus, and osteoarthritis.

\section{References}

1. Colledge NR. Ageing and disease. In: Boon NA, Colledge NR, Walker BR, et al., editors. Davidsons text book of medicine. London; 2006. p. 64-71.

2. Clegg A, Young J, Iliffe $S$, et al. Frailty in elderly people. Lancet 2013;381(9868):752-762. DOI: 10.1016/S0140-6736(12)62167-9.

3. World Health Organization. WHO clinical consortium on healthy ageing: topic focus: frailty and intrinsic capacity: report of consortium meeting, 1-2 December 2016 in Geneva, Switzerland. Geneva, Switzerland: World Health Organization; 2017.

4. Fried LP, Tangen CM, Walston J, et al. Frailty in older adults: evidence for a phenotype. J Gerontol A BiolSci Med Sci 2001;56(3):M146-M156. DOI: $10.1093 /$ gerona/56.3.m146.

5. De Martinis M, Franceschi C, Monti D, et al. Inflammation markers predicting frailty and mortality in the elderly. Exp Mol Pathol 2006;80(3):219-227. DOI: 10.1016/j.yexmp.2005.11.004..

6. Hertoghe T. The "multiple hormone deficiency" theory of aging: is human senescence caused mainly by multiple hormone deficiencies? Ann N Y Acad Sci 2005;1057:448-465. DOI: 10.1196/ annals.1322.035.

7. Leng $\mathrm{S}, \mathrm{Chaves} \mathrm{P}$, Koenig $\mathrm{K}$, et al. Serum interleukin- 6 and hemoglobin as physiological correlates in the geriatric syndrome of frailty: a pilot study. J Am Geriatr Soc 2002;50(7):1268-1271. DOI: 10.1046/ j.1532-5415.2002.50315.x.

8. Roubenoff R. Molecular basis of inflammation: relationships between catabolic cytokines, hormones, energy balance, and muscle. JPEN J Parenter Enteral Nutr 2008;32(6):630-632. DOI: $10.1177 / 0148607108324875$.

9. Qu T, Yang H, Walston JD, et al. Upregulated monocytic expression of CXC chemokine ligand 10 (CXCL-10) and its relationship with serum interleukin- 6 levels in the syndrome of frailty. Cytokine.2009;46(3):319-324. DOI: 10.1016/j.cyto.2009.02.015.

10. Hubbard RE, O'Mahony MS, Calver BL, et al. Plasma esterases and inflammation in ageing and frailty. Eur J Clin Pharmacol 2008;64(9):895-900. DOI: 10.1007/s00228-008-0499-1.

11. Leng SX, Xue QL, Tian J, et al. Associations of neutrophil and monocyte counts with frailty in community-dwelling disabled older women: results from the Women's Health and Aging Studies I. Exp Gerontol 2009;44(8):511-516. DOI: 10.1016/j.exger.2009.05.005.

12. Leng SX, Xue QL, Tian J, et al. Inflammation and frailty in older women. J Am Geriatr Soc 2007;55(6):864-871. DOI: 10.1111/j.15325415.2007.01186.x.

13. Hajek A, Bock JO, Saum KU, et al. Frailty and healthcare costslongitudinal results of a prospective cohort study. Age Ageing 2018;47(2):233-241. DOI: 10.1093/ageing/afx157.

14. Bock JO, König $\mathrm{HH}$, Brenner $\mathrm{H}$, et al. Associations of frailty with health care costs - results of the ESTHER cohort study. BMC Health Serv Res 2016;16(1):128. DOI: 10.1186/s12913-016-1360-3.

15. Salinas-Rodriguez A, Manrique-Espinoza B, Heredia-Pi I, et al. Healthcare costs of frailty: implications for long-term care. J Am Med Dir Assoc 2019;20(1):102-103.e2. DOI: 10.1016/j.jamda.2018.09.019.

16. Ensrud KE, Kats AM, Schousboe JT, et al. Frailty phenotype and healthcare costs and utilization in older women. J Am Geriatr Soc 2018;66(7):1276-1283. DOI: 10.1111/jgs.15381.

17. Simpson KN, Seamon BA, Hand BN, et al. Effect of frailty on resource use and cost for Medicare patients. J Comp Eff Res 2018;7(8):817-825. DOI: 10.2217/cer-2018-0029. 
18. Mulasso A, Roppolo M, Rabaglietti E. Physical frailty, disability, and dynamics in health perceptions: a preliminary mediation model. Clin Interv Aging 2016;11:275-278. DOI: 10.2147/CIA.S97507.

19. de Labra C, Maseda A, Lorenzo-Lopez L, et al. Social factors and quality of life aspects on frailty syndrome in community-dwelling older adults: the verisaude study. BMC Geriatr 2018;18(1):66. DOI: 10.1186/s12877-018-0757-8.

20. Chang SF, Lin PL. Frail phenotype and mortality prediction: a systematic review and meta-analysis of prospective cohort studies. Int J Nurs Stud 2015;52(8):1362-1374. DOI: 10.1016/j.ijnurstu.2015.04.005.

21. Avila-Funes JA, Amieva H, Barberger-Gateau P, et al. Cognitive impairment improves the predictive validity of the phenotype of frailty for adverse health outcomes: the three-city study. J Am Geriatr Soc 2009;57(3):453-461. DOI: 10.1111/j.1532-5415.2008.02136.x.

22. Santos-Eggimann B, Cuenoud P, Spagnoli J, et al. Prevalence of frailty in middle-aged and older community-dwelling Europeans living in 10 countries. J Gerontol A Biol Sci Med Sci 2009;64(6):675-681. DOI: 10.1093/gerona/glp012.

23. Alvarado $\mathrm{BE}$, Zunzunegui MV, Beland F, et al. Life course social and health conditions linked to frailty in Latin American older men and women. J Gerontol A Biol Sci Med Sci 2008;63(12):1399-1406. DOI: 10.1093/gerona/63.12.1399.

24. Bandeen-Roche K, Xue QL, Ferrucci L, et al. Phenotype of frailty: characterization in the women's health and aging studies. J Gerontol A BiolSci Med Sci 2006;61(3):262-266. DOI: 10.1093/gerona/61.3.262.

25. Hogan DB, MacKnight C, Bergman H, Steering Committee CloFaA. Models, definitions, and criteria of frailty. Aging Clin Exp Res 2003;15(3 Suppl.):1-29. DOI: 10.1016/b978-0-12-811353-0.00003-8.

26. Ensrud KE, Ewing SK, Taylor BC, et al. Comparison of 2 frailty indexes for prediction of falls, disability, fractures, and death in older women. Arch Intern Med 2008;168(4):382-389. DOI: 10.1001/ archinternmed.2007.113.

27. Sarkisian CA, Gruenewald TL, John Boscardin W, et al. Preliminaryevidence for sub-dimensions of geriatric frailty: the MacArthur study ofsuccessful aging. J Am Geriatr Soc 2008;56(12):2292-2297. DOI: 10.1111/j.1532-5415.2008.02041.x.

28. Walston J, Hadley EC, Ferrucci L, et al. Research agenda for frailty in older adults: toward a better understanding of physiology and etiology: summary from the American Geriatrics Society/ National Institute on Aging Research Conference on Frailty in Older Adults. J Am Geriatr Soc 2006;54(6):991-1001. DOI: 10.1111/j.15325415.2006.00745.x.

29. Varadhan R, Seplaki CL, Xue QL, et al. Stimulus-response paradigm for characterizing the loss of resilience in homeostatic regulation associated with frailty. Mech Ageing 2008;129:666-670. DOI: 10.1016/j.mad.2008.09.013.

30. Boyd CM, Xue QL, Simpson CF, et al. Frailty, hospitalization, and progression of disability in a cohort of disabled older women. Am J Med 2005;118(11):1225-1231. DOI: 10.1016/j.amjmed.2005.01.062.

31. Taylor R. Measuring healthy days, population assessment of healthrelated quality of life. CDC; 2000.

32. Cigolle CT, Lee PG, Langa KM, et al. Geriatric conditions develop in middle-aged adults with diabetes. J Gen Intern Med 2011;26(3): 272-279. DOI: 10.1007/s11606-010-1510-y.

33. Tsou IY, Ching HH. The bone and joint decade 2000-2010 for prevention and treatment of musculoskeletal disease. Ann Acad Med Singap 2002;31(1):69-70. PMID: 11885499.

34. Graham JE, Snih SA, Berges IM, et al. Frailty and 10-year mortality in community-living Mexican American older adults. Gerontology 2009:55(6):644-651. DOI: 10.1159/000235653. 